\title{
The heat flow for the full bosonic string
}

\author{
Volker Branding ${ }^{1}$
}

Received: 5 November 2015 / Accepted: 6 May 2016 / Published online: 31 May 2016

(C) The Author(s) 2016. This article is published with open access at Springerlink.com

\begin{abstract}
We study harmonic maps from surfaces coupled to a scalar and a two-form potential, which arise as critical points of the action of the full bosonic string. We investigate several analytic and geometric properties of these maps and prove an existence result by the heat-flow method.
\end{abstract}

Keywords Harmonic maps with scalar and two-form potential · Full bosonic string · Heat flow

Mathematics Subject Classification 58E20 - 35K55 - 53C08 · 53C80

\section{Introduction and results}

Harmonic maps between Riemannian manifolds are one of the most studied variational problems in differential geometry. Under the assumption that the target manifold has nonpositive curvature Eells and Sampson established their famous existence result for harmonic maps making use of the heat-flow method [10]. Moreover, in the case that the domain is twodimensional harmonic maps belong to the class of conformally invariant variational problems yielding a rich structure.

However, harmonic maps from surfaces also have a dual life in theoretical physics. More precisely, they arise as the Polyakov action in bosonic string theory. The full action for the bosonic string contains two additional terms, one of them being the pullback of a two-form from the target and the other one being a scalar potential. It is the aim of this article to study the full action of the bosonic string as a geometric variational problem.

There are already several mathematical results available for parts of the energy functional of the full bosonic string: in [11] the authors consider the harmonic map energy together with

Volker Branding

volker@geometrie.tuwien.ac.at

1 Institut für diskrete Mathematik und Geometrie, TU Wien, Wiedner Hauptstrasse 8-10, 1040 Vienna, Austria 
a scalar potential. The critical points of this energy functional are called harmonic maps with potential. One of the main results in that reference is that depending on the choice of potential, the qualitative behavior of harmonic maps with potential differs from the one of harmonic maps. There are several results available that characterize the properties of harmonic maps with potential: this includes gradient estimates [6] and Liouville theorems [7] for harmonic maps with potential from complete manifolds. In [8] an existence result for harmonic maps with potential from compact Riemannian manifolds with boundary is obtained, where it is assumed that the image of the map lies inside a convex ball. An existence result for harmonic maps with potential to a target with negative curvature was obtained in [12] by the heat-flow method. This result has been extended to the case of a domain manifold with boundary in [9]. Harmonic maps from surfaces coupled to a two-form potential have also been studied in the mathematical literature since they give rise to the prescribed mean curvature equation. Existence results via the heat flow for the prescribed mean curvature equation have been obtained for a flat target in [23] and for a three-dimensional target with negative curvature in [27].

In theoretical physics the two-form potential is interpreted as giving rise to an external magnetic field.

Harmonic maps coupled to a two-form potential and spinor fields instead of a scalar potential have been studied in [4].

We will call the critical points of the full bosonic string action harmonic maps with scalar and two-form potential and we will generalize several results already obtained for harmonic maps and harmonic maps with potential. Moreover, we will point out new phenomena that arise from the two-form potential.

This article is organized as follows. In Sect. 2, we analyze the energy functional of the full bosonic string and derive its critical points. In Sect. 3, we study analytic and geometric aspects of the critical points. In the last section, we derive an existence result via the heat-flow method for both compact and non-compact target manifolds.

\section{The full bosonic string action}

Throughout this article $(M, h)$ is a Riemannian surface without boundary, we will mostly assume that $M$ is compact, and $(N, g)$ a closed, oriented Riemannian manifold of dimension $\operatorname{dim} N \geq 3$. For a map $\phi: M \rightarrow N$ we consider the square of its differential giving rise to the usual harmonic energy. Let $B$ be a two-form on $N$, which we pull back by the map $\phi$. In addition, let $V: N \rightarrow \mathbb{R}$ be a scalar function, which we mostly assume to be smooth. By $R$ we denote the scalar curvature of the domain $M$.

In the physical literature the full action for the bosonic string is given by

$$
E(\phi)=\int_{M}\left(\frac{1}{2}|\mathrm{~d} \phi|^{2}+\phi^{*} B+R V(\phi)\right) \mathrm{d} M,
$$

see for example [22, p. 108]. Due to the uniformization theorem we can assume that the scalar curvature $R$ on the domain $M$ is constant. In string theory, the potential $V(\phi)$ is usually referred to as dilaton field.

For the sake of completeness, we want to mention that in theoretical physics one also defines an action functional that locally has the form (2.1) but has a different global structure. More precisely, one replaces the two-form contribution in (2.1) by an object that locally looks 
like a two-form, but forms a more general object from the global point of view. One then studies the $U(1)$-valued functional

$$
\exp (i E(\phi)):=\exp \left(i \int_{M}\left(\frac{1}{2}|\mathrm{~d} \phi|^{2}+R V(\phi)\right) \mathrm{d} M\right) \cdot \operatorname{hol}(\phi),
$$

where $\operatorname{hol}(\phi)$ denotes the holonomy of the gerbe $\mathcal{G}$ along the map $\phi$. By the three-form $\Omega$ we denote the globally defined curvature associated to the gerbe $\mathcal{G}$. For a mathematical introduction to gerbes and surface holonomy see [13]. This gives rise to the so-called $B$-field action in string theory, which is defined as

$$
E_{B}(\phi):=-i \log (\operatorname{hol}(\phi))
$$

such that $\operatorname{hol}(\phi)=\exp \left(i E_{B}(\phi)\right)$.

In the case that the gerbe $\mathcal{G}$ is trivial, which corresponds to the fact that the three-form $\Omega$ is exact, we can define the $B$-field action as

$$
E_{B}(\phi):=\int_{M} \phi^{*} B \mathrm{~d} M
$$

leading to the functional (2.1). From an analytical point of view the $U(1)$-valued functional (2.2) is more difficult, and we will restrict ourselves to the functional (2.1).

Let us derive the critical points of (2.1).

Proposition 2.1 The Euler-Lagrange equation of the functional (2.1) is given by

$$
\tau(\phi)=Z\left(\mathrm{~d} \phi\left(e_{1}\right) \wedge \mathrm{d} \phi\left(e_{2}\right)\right)+R \nabla V(\phi),
$$

where $\tau(\phi)$ denotes the tension field of the map $\phi$ and the vector-bundle homomorphism $Z \in \Gamma\left(\operatorname{Hom}\left(\Lambda^{2} T^{*} N, T N\right)\right)$ is defined by the equation

$$
\Omega\left(\eta, \xi_{1}, \xi_{2}\right)=\left\langle Z\left(\xi_{1} \wedge \xi_{2}\right), \eta\right\rangle,
$$

where $\Omega=\mathrm{d} B$ is a three-form on $N$ and $e_{1}, e_{2}$ an orthonormal basis of $T M$.

Proof We consider a smooth variation of $\phi$ that is $\phi_{t}:(-\epsilon, \epsilon) \times M \rightarrow N$ with $\left.\frac{\partial \phi_{t}}{\partial t}\right|_{t=0}=\eta$. It is well-known that

$$
\begin{aligned}
& \left.\frac{\mathrm{d}}{\mathrm{d} t}\right|_{t=0} \frac{1}{2} \int_{M}\left|\mathrm{~d} \phi_{t}\right|^{2} \mathrm{~d} M=-\int_{M}\langle\tau(\phi), \eta\rangle \mathrm{d} M, \\
& \left.\frac{\mathrm{d}}{\mathrm{d} t}\right|_{t=0} \int_{M} R V\left(\phi_{t}\right) \mathrm{d} M=\int_{M} R\langle\nabla V(\phi), \eta\rangle \mathrm{d} M .
\end{aligned}
$$

To calculate the variation of the two-form $B$, we choose an orthonormal basis $e_{1}, e_{2}$ of $T M$ and calculate

$$
\begin{aligned}
\frac{\mathrm{d}}{\mathrm{d} t}\left(\phi_{t}^{*} B\right)\left(e_{1}, e_{2}\right) & =\phi_{t}^{*}\left(\mathcal{L}_{\partial_{t}} B\right)\left(e_{1}, e_{2}\right) \\
& =\phi_{t}^{*}\left(\iota_{\partial_{t}} \mathrm{~d} B\left(e_{1}, e_{2}\right)+d\left(\iota_{\partial_{t}} B\left(e_{1}, e_{2}\right)\right)\right) \\
& =\phi_{t}^{*}\left(\Omega\left(\partial_{t}, e_{1}, e_{2}\right)\right)+d\left(\phi_{t}^{*}\left(\iota_{\partial_{t}} B\left(e_{1}, e_{2}\right)\right)\right),
\end{aligned}
$$

where we used that $\mathrm{d} B=\Omega$. Here, $\mathcal{L}$ denotes the Lie-derivative applied to differential forms. Moreover, we applied several identities for derivatives of differential forms, which can be found in [24, pp. 170-174]. 
Integrating over $M$ we thus obtain

$$
\left.\frac{\mathrm{d}}{\mathrm{d} t}\right|_{t=0} \int_{M}\left(\phi_{t}^{*} B\right)\left(e_{1}, e_{2}\right) \mathrm{d} M=\int_{M} \Omega\left(\eta, \mathrm{d} \phi\left(e_{1}\right), \mathrm{d} \phi\left(e_{2}\right)\right) \mathrm{d} M .
$$

Using the vector-bundle homomorphism (2.4), we thus find

$$
\left.\frac{\mathrm{d}}{\mathrm{d} t}\right|_{t=0} E\left(\phi_{t}\right)=\int_{M}\left\langle\eta,-\tau(\phi)+Z\left(\mathrm{~d} \phi\left(e_{1}\right) \wedge \mathrm{d} \phi\left(e_{2}\right)\right)+R \nabla V(\phi)\right\rangle \mathrm{d} M
$$

yielding the claim.

We call solutions of (2.3) harmonic maps with scalar and two-form potential.

Remark 2.2 Note that the energy functionals (2.1) and (2.2) have the same critical points since

$$
\left.\frac{\mathrm{d}}{\mathrm{d} t}\right|_{t=0} \exp \left(i E\left(\phi_{t}\right)\right)=\left.0 \Leftrightarrow \frac{\mathrm{d}}{\mathrm{d} t}\right|_{t=0} \int_{M}\left(\frac{1}{2}\left|\mathrm{~d} \phi_{t}\right|^{2}+R V\left(\phi_{t}\right)\right) \mathrm{d} M+\left.\frac{\mathrm{d}}{\mathrm{d} t}\right|_{t=0} E_{B}\left(\phi_{t}\right)=0 .
$$

Whenever using local coordinates, we will use Greek indices on the domain $M$ and Latin indices on the target $N$. Moreover, we will make use of the Einstein summation convention, that is, we sum over repeated indices. In terms of local coordinates $x_{\alpha}$ on $M$ and $y^{i}$ on $N$ the Euler-Lagrange equation reads

$$
\Delta \phi^{i}=-\Gamma_{j k}^{i} \frac{\partial \phi^{j}}{\partial x_{\alpha}} \frac{\partial \phi^{k}}{\partial x_{\beta}} h_{\alpha \beta}+Z^{i}\left(\partial_{y^{j}} \wedge \partial_{y^{k}}\right) \frac{\partial \phi^{j}}{\partial x_{1}} \frac{\partial \phi^{k}}{\partial x_{2}}+R g^{i k} \frac{\partial V}{\partial y^{k}} .
$$

Remark 2.3 The vector-bundle homomorphism $Z$ can also be interpreted as arising from a metric connection with totally antisymmetric torsion. In this case one has

$$
\nabla_{X}^{T o r} Y=\nabla_{X}^{L C} Y+A(X, Y),
$$

where $\nabla^{L C}$ denotes the Levi-Cevita connection, $X, Y$ are vector fields and $A(X, Y)$ is a skew-adjoint endomorphism. The endomorphism $A(X, Y)$ satisfies

$$
\langle A(X, Y), Z\rangle=\Omega(X, Y, Z)
$$

with $\Omega \in \Gamma\left(\Lambda^{3} T^{*} N\right)$ similar to (2.4). For more details on metric connections with torsion see [3] and references therein.

Remark 2.4 In principle one could also study the functional (2.1) for a higher-dimensional domain $M$ with $m=\operatorname{dim} M \geq 2$. However, then one needs to pull back an $m$-form from the target leading to an Euler-Lagrange equation with a higher nonlinearity on the right hand side, see [17, Chapter 2], for a detailed analysis.

Lemma 2.5 (Second variation) Let $\phi: M \rightarrow N$ be a harmonic map with scalar and twoform potential. The second variation of the energy functional (2.1) is given by

$$
\begin{aligned}
& \left.\frac{\partial^{2}}{\partial t^{2}}\right|_{t=0} E\left(\phi_{t}\right)=\int_{M}\left(|\nabla \xi|^{2}-\left\langle R^{N}(\mathrm{~d} \phi, \xi) \mathrm{d} \phi, \xi\right\rangle+\left\langle\xi,\left(\nabla_{\xi} Z\right)\left(\mathrm{d} \phi\left(e_{1}\right) \wedge \mathrm{d} \phi\left(e_{2}\right)\right)\right\rangle\right. \\
& \left.\quad+\left\langle\xi, Z\left(\nabla \xi\left(e_{1}\right) \wedge \mathrm{d} \phi\left(e_{2}\right)\right)\right\rangle+\left\langle\xi, Z\left(\mathrm{~d} \phi\left(e_{1}\right) \wedge \nabla \xi\left(e_{2}\right)\right)\right\rangle+R \operatorname{Hess} V(\xi, \xi)\right) \mathrm{d} M
\end{aligned}
$$

where $\xi=\left.\frac{\partial \phi_{t}}{\partial t}\right|_{t=0}$.

Proof This follows by a direct calculation. 
Example 2.6 Suppose that $\phi$ is an isometric immersion from a closed oriented Riemann surface $(M, h)$ into an oriented Riemannian three manifold $(N, g)$. In this case the tension field $\phi$ is related to the mean curvature vector $H(\phi)$ by

$$
\frac{1}{2} \tau(\phi)=H(\phi)
$$

Any three-form $\Omega$ on $N$ must be a multiple of the volume form $\operatorname{vol}_{g}$, that is

$$
\Omega=f \operatorname{vol}_{g}
$$

for some smooth function $f: N \rightarrow \mathbb{R}$. Thus, if we denote the unit normal field of $\phi(M) \subset N$ by $v$ the Euler-Lagrange equation (2.3) is equivalent to

$$
2 H(\phi)= \pm(f \nu) \circ \phi+R \nabla V(\phi) .
$$

The sign in front of the first term on the right hand side depends on whether the map $\phi$ is orientation preserving or not, see [17, Remark 2.7].

In case that the function $f$ is constant and $\nabla V \sim v$ the equation (2.7) has some similarity with the equation for linear Weingarten surfaces. These are surfaces immersed in a threedimensional manifold satisfying

$$
a H+b K=c,
$$

where $H$ denotes the mean curvature, $K$ the Gauss curvature and $a, b, c$ are non-zero real numbers.

\section{Properties of harmonic maps with scalar and two-form potential}

In this section we study several properties of solutions of (2.3).

Lemma 3.1 Let $\phi \in C^{2}(M, N)$ be a solution of (2.3). Then the following formulas hold:

$$
\begin{aligned}
\Delta \frac{1}{2}|\mathrm{~d} \phi|^{2}= & |\nabla \mathrm{d} \phi|^{2}-\left\langle R^{N}\left(\mathrm{~d} \phi\left(e_{\alpha}\right), \mathrm{d} \phi\left(e_{\beta}\right)\right) \mathrm{d} \phi\left(e_{\alpha}\right), \mathrm{d} \phi\left(e_{\beta}\right)\right\rangle+\left\langle\mathrm{d} \phi\left(\operatorname{Ric}^{M}\left(e_{\alpha}\right)\right), \mathrm{d} \phi\left(e_{\alpha}\right)\right\rangle \\
& -\left\langle Z\left(\mathrm{~d} \phi\left(e_{1}\right) \wedge \mathrm{d} \phi\left(e_{2}\right)\right), \tau(\phi)\right\rangle+R \operatorname{Hess} V(\mathrm{~d} \phi, \mathrm{d} \phi)
\end{aligned}
$$

and

$$
\Delta(V \circ \phi)=\mathrm{d} V\left(R \nabla V(\phi)+Z\left(\mathrm{~d} \phi\left(e_{1}\right) \wedge \mathrm{d} \phi\left(e_{2}\right)\right)\right)+\operatorname{Tr} \operatorname{Hess} V(\mathrm{~d} \phi, \mathrm{d} \phi),
$$

where $\operatorname{Ric}^{M}$ denotes the Ricci-curvature on $M$ and $R^{N}$ the curvature tensor on $N$.

Proof This follows by a direct calculation.

Definition 3.2 We define a two-tensor by

$$
S_{\alpha \beta}:=\frac{1}{2} h_{\alpha \beta}|\mathrm{d} \phi|^{2}-\left\langle\mathrm{d} \phi\left(e_{\alpha}\right), \mathrm{d} \phi\left(e_{\beta}\right)\right\rangle+R V(\phi) h_{\alpha \beta} .
$$

The tensor $S_{\alpha \beta}$ is called the stress-energy tensor.

Lemma 3.3 The stress-energy tensor $S_{\alpha \beta}$ is divergence free and symmetric if $\phi$ is a harmonic map with scalar and two-form potential. 
Proof By a direct calculation we find

$$
\begin{aligned}
\nabla_{e_{\alpha}} S_{\alpha \beta} & =-\left\langle\tau(\phi), \mathrm{d} \phi\left(e_{\beta}\right)\right\rangle+\left\langle R \nabla V(\phi), \mathrm{d} \phi\left(e_{\beta}\right)\right\rangle \\
& =-\left\langle Z\left(\mathrm{~d} \phi\left(e_{1}\right) \wedge \mathrm{d} \phi\left(e_{2}\right)\right), \mathrm{d} \phi\left(e_{\beta}\right)\right\rangle \\
& =-\Omega\left(\mathrm{d} \phi\left(e_{1}\right), \mathrm{d} \phi\left(e_{2}\right), \mathrm{d} \phi\left(e_{\beta}\right)\right)=0
\end{aligned}
$$

which proves the claim.

Note that $S_{\alpha \beta}$ is no longer trace-free, which corresponds to the fact that the scalar potential $V(\phi)$ in the action functional does not respect the conformal symmetry.

We can express the stress-energy tensor (3.3) invariantly as

$$
S_{V}(\phi)=e(\phi) h-\phi^{*} g+R V(\phi) h
$$

with the energy density $e(\phi)=\frac{1}{2}|\mathrm{~d} \phi|^{2}$.

With the help of (3.4) we now derive a monotonicity formula for $e(\phi)+R V(\phi)$. A similar calculation for harmonic maps with scalar potential has been carried out in [20]. For the monotonicity formula for harmonic maps we refer to the book [28], for a general treatment of stress-energy tensors with applications to harmonic maps we refer to [2]. Note that we do not have to assume that $M$ is compact to derive the monotonicity formula.

Let $\left(M, h_{0}\right)$ be a complete Riemannian surface with a pole $x_{0}$. Let $r(x)$ be the Riemannian distance function relative to the point $x_{0}$. By $\lambda_{i}, i=1,2$ we denote the eigenvalues of $\operatorname{Hess}_{h_{0}}\left(r^{2}\right)$.

Proposition 3.4 (Monotonicity formula) Let $\phi:\left(M, f^{2} h_{0}\right) \rightarrow(N, g)$ be a harmonic map with scalar and two-form potential, where $M$ is a complete Riemannian surface and $N a$ Riemannian manifold. Suppose that

$$
R V(\phi)>0, \quad r \frac{\partial \log f}{\partial r} \geq 0, \quad \frac{1}{2}\left(\sum_{i=1}^{2} \lambda_{i}-2 \lambda_{\max }\right) \geq \sigma
$$

for a positive constant $\sigma$. Then the following inequality holds

$$
\frac{\int_{B_{\rho_{1}}\left(x_{0}\right)}(e(\phi)+R V(\phi))}{\rho_{1}^{\sigma}} \leq \frac{\int_{B_{\rho_{2}}\left(x_{0}\right)}(e(\phi)+R V(\phi))}{\rho_{2}^{\sigma}}
$$

for any $0<\rho_{1} \leq \rho_{2}$. Here, $B_{\rho}(x)$ denotes the geodesic ball with radius $\rho$ around the point $x$.

The proof is similar to the proof of Theorem 4.1 in [20].

Proof For a symmetric (2, 0)-tensor $S$ the following identity holds

$$
\operatorname{div}\left(\iota_{X} S\right)=(\operatorname{div} S)(X)+\frac{1}{2}\left\langle S, \mathcal{L}_{X} h\right\rangle .
$$

Here, $\mathcal{L}_{X}$ represents the Lie-derivative with respect to the vector field $X$. Integrating the divergence of (3.4) over the ball $B_{\rho}\left(x_{0}\right)$ with radius $\rho$ around the point $x_{0}$, using (3.7) and the fact that the stress-energy-tensor is divergence free, we obtain

$$
\int_{\partial B_{\rho}\left(x_{0}\right)} S_{V}(\phi)(X, v)=\frac{1}{2} \int_{B_{\rho}\left(x_{0}\right)}\left\langle S_{V}(\phi), \mathcal{L}_{X} h\right\rangle .
$$


We choose $X=r \frac{\partial}{\partial r}$ and get

$$
\frac{1}{2} \mathcal{L}_{X} h=\frac{1}{2} \mathcal{L}_{X}\left(f^{2} h_{0}\right)=r f \frac{\partial f}{\partial r} h_{0}+\frac{1}{2} f^{2} \mathcal{L}_{X} h_{0},
$$

where we used that the metric on $M$ is given by $h=f^{2} h_{0}$. Hence, we find

$$
\frac{1}{2}\left\langle S_{V}(\phi), \mathcal{L}_{X} h\right\rangle=2 R V(\phi) r \frac{\partial \log f}{\partial r}+\frac{1}{2} f^{2}\left\langle S_{V}(\phi), \operatorname{Hess}_{h_{0}}\left(r^{2}\right)\right\rangle
$$

since $\operatorname{dim} M=2$.

To estimate the last term on the right hand side, we choose a local basis $e_{1}, e_{2}$ on $M$ with respect to $h_{0}$ such that $\operatorname{Hess}_{h_{0}}\left(r^{2}\right)$ becomes a diagonal matrix with respect to $e_{1}, e_{2}$. Then $\tilde{e}_{\alpha}:=f^{-1} e_{\alpha}, \alpha=1,2$ is an orthonormal basis with respect to $h$. Thus, we obtain

$$
\begin{aligned}
& f^{2}\left\langle S_{V}(\phi), \operatorname{Hess}_{h_{0}}\left(r^{2}\right)\right\rangle=f^{2} \sum_{\alpha, \beta=1}^{2} S_{V}(\phi)\left(\tilde{e}_{\alpha}, \tilde{e}_{\beta}\right) \operatorname{Hess}_{h_{0}}\left(r^{2}\right)\left(\tilde{e}_{\alpha}, \tilde{e}_{\beta}\right) \\
& \quad=\sum_{\alpha, \beta=1}^{2}\left(e(\phi) h\left(\tilde{e}_{\alpha}, \tilde{e}_{\beta}\right)-\phi^{*} g\left(\tilde{e}_{\alpha}, \tilde{e}_{\beta}\right)+R V(\phi) g\left(\tilde{e}_{\alpha}, \tilde{e}_{\beta}\right)\right) \operatorname{Hess}_{h_{0}}\left(r^{2}\right)\left(e_{\alpha}, e_{\beta}\right) \\
& =\sum_{\alpha=1}^{2}(e(\phi)+R V(\phi)) \operatorname{Hess}_{h_{0}}\left(r^{2}\right)\left(e_{\alpha}, e_{\alpha}\right)-\sum_{\alpha=1}^{2} \phi^{*} g\left(\tilde{e}_{\alpha}, \tilde{e}_{\alpha}\right) \operatorname{Hess}_{h_{0}}\left(r^{2}\right)\left(e_{\alpha}, e_{\alpha}\right) \\
& \geq(e(\phi)+R V(\phi)) \sum_{i=1}^{2} \lambda_{i}-2 e(\phi) \lambda_{\max } \\
& \geq(e(\phi)+R V(\phi))\left(\Lambda-2 \lambda_{\max }\right),
\end{aligned}
$$

where $\Lambda=\lambda_{1}+\lambda_{2}$. Hence, we obtain

$$
\frac{1}{2}\left\langle S_{V}(\phi), \mathcal{L}_{x} h\right\rangle \geq 2 R V(\phi) r \frac{\partial \log f}{\partial r}+\frac{1}{2}(e(\phi)+R V(\phi))\left(\Lambda-2 \lambda_{\max }\right) .
$$

On the other hand, by the coarea formula and $|\nabla r|=f^{-1}$ we find

$$
\begin{aligned}
\int_{\partial B_{\rho}\left(x_{0}\right)} S_{V}(\phi)\left(r \frac{\partial}{\partial r}, v\right) & \leq \int_{\partial B_{\rho}\left(x_{0}\right)}(e(\phi)+R V(\phi)) h\left(r \frac{\partial}{\partial r}, v\right) \\
& =\rho \int_{\partial B_{\rho}\left(x_{0}\right)}(e(\phi)+R V(\phi)) f \\
& =\rho \frac{\mathrm{d}}{\mathrm{d} \rho} \int_{B_{\rho}\left(x_{0}\right)}\left(\int_{\partial B_{r}\left(x_{0}\right)} \frac{e(\phi)+R V(\phi)}{|\nabla r|}\right) \mathrm{d} r \\
& =\rho \frac{\mathrm{d}}{\mathrm{d} \rho} \int_{B_{\rho}\left(x_{0}\right)}(e(\phi)+R V(\phi)) .
\end{aligned}
$$

Thus, by the assumptions (3.5) we obtain the following inequality

$$
\rho \frac{\mathrm{d}}{\mathrm{d} \rho} \int_{B_{\rho}\left(x_{0}\right)}(e(\phi)+R V(\phi)) \geq \int_{B_{\rho}\left(x_{0}\right)} \frac{1}{2}(e(\phi)+R V(\phi))\left(\Lambda-2 \lambda_{\max }\right) .
$$

Again, by assumption we get

$$
\rho \frac{\mathrm{d}}{\mathrm{d} \rho} \int_{B_{\rho}\left(x_{0}\right)}(e(\phi)+R V(\phi)) \geq \sigma \int_{B_{\rho}\left(x_{0}\right)}(e(\phi)+R V(\phi)) .
$$


This can be rewritten as

$$
\frac{\mathrm{d}}{\mathrm{d} \rho} \frac{\int_{B_{\rho}\left(x_{0}\right)}(e(\phi)+R V(\phi))}{\rho^{\sigma}} \geq 0
$$

and the claim follows by integration with respect to $\rho$.

As for harmonic maps ([25, Theorem 2]) and harmonic maps with potential ([11, Proposition 2]) we can prove a unique continuation theorem for harmonic maps with scalar and two-form potential. To obtain this result we recall the following ([1, p. 248])

Theorem 3.5 Let $A$ be a linear elliptic second-order differential operator defined on a domain $D$ of $\mathbb{R}^{n}$. Let $u=\left(u^{1}, \ldots, u^{n}\right)$ be functions in $D$ satisfying the inequality

$$
\left|A u^{j}\right| \leq C\left(\sum_{\alpha, i}\left|\frac{\partial u^{i}}{\partial x^{\alpha}}\right|+\sum_{i}\left|u^{i}\right|\right) .
$$

If $u=0$ in an open set, then $u=0$ throughout $D$.

Making use of this result we can prove the following

Proposition 3.6 Let $\phi, \phi^{\prime} \in C^{2}(M, N)$ be two harmonic maps with scalar and two-form potential. Moreover, assume that $V: N \rightarrow \mathbb{R}$ is a $C^{1,1}$ function. If $\phi$ and $\phi^{\prime}$ are equal on a connected open set $W$ of $M$ then they coincide on the whole connected component of $M$ which contains $W$.

Proof Let $U$ be a coordinate ball on $M$ such that $\phi=\phi^{\prime}$ in some open subset. By shrinking $U$ if necessary we can assume that both $\phi$ and $\phi^{\prime}$ map $U$ into a single coordinate chart in $N$. We write $y^{i}(x)$ for $y^{i}(\phi(x))$ and $z^{i}(x)$ for $z^{i}\left(\phi^{\prime}(x)\right)$. We consider the function $u^{i}:=y^{i}-z^{i}$. Using the local form of the Euler-Lagrange equation (2.5) we find

$$
\begin{aligned}
\Delta u^{i}= & -\Gamma_{j k}^{i}(y) \frac{\partial y^{j}}{\partial x_{\alpha}} \frac{\partial y^{k}}{\partial x_{\beta}} h_{\alpha \beta}+\Gamma_{j k}^{i}(z) \frac{\partial z^{j}}{\partial x_{\alpha}} \frac{\partial z^{k}}{\partial x_{\beta}} h_{\alpha \beta} \\
& -Z^{i}(y)\left(\partial_{y^{j}} \wedge \partial_{y^{k}}\right) \frac{\partial y^{j}}{\partial x_{1}} \frac{\partial y^{k}}{\partial x_{2}}+Z^{i}(z)\left(\partial_{y^{j}} \wedge \partial_{y^{k}}\right) \frac{\partial z^{j}}{\partial x_{1}} \frac{\partial z^{k}}{\partial x_{2}} \\
& +R g^{i k}(y) \frac{\partial V}{\partial y^{k}}(y)-\operatorname{Rg}^{i k}(z) \frac{\partial V}{\partial y^{k}}(z) .
\end{aligned}
$$

By adding several zero's on the right hand side the above equation acquires the form (3.8) and the result follows by application of Theorem 3.5. For more details see the proof of Theorem 2 in [25].

Remark 3.7 In the case that the scalar potential $V(\phi)$ vanishes identically, the energy functional (2.1) is conformally invariant. In this case one can exploit the conformal invariance to prove that critical points cannot have isolated singularities, whenever a certain energy is finite. This follows from the main theorem in [14], where a removable singularity theorem for critical points of a large class of conformally invariant energy functionals is established.

As a next step, we use the embedding theorem of Nash to isometrically embed $N$ into some $\mathbb{R}^{q}$. We denote the isometric embedding by $\iota$ and consider the composite map $u:=$ $\iota \circ: M \rightarrow \mathbb{R}^{q}$. By $\tilde{N}$ we denote the tubular neighborhood of $\iota(N) \subset \mathbb{R}^{q}$. Let $\pi: \tilde{N} \rightarrow \iota(N)$ be the canonical projection which assigns to each $z \in \tilde{N}$ the closest point in $\iota(N)$ from $z$. 
Lemma 3.8 Assume that $N \subset \mathbb{R}^{q}$. Then the Euler-Lagrange equation (2.3) acquires the form

$$
\Delta u=\mathbb{I}(\mathrm{d} u, \mathrm{~d} u)+\tilde{Z}\left(\mathrm{~d} u\left(e_{1}\right) \wedge \mathrm{d} u\left(e_{2}\right)\right)+\widetilde{R \nabla V}(u),
$$

where $\mathbb{I}(\mathrm{d} u, \mathrm{~d} u):=\operatorname{Tr} \nabla \mathrm{d} \pi(\mathrm{d} u, \mathrm{~d} u)$. Moreover, $\tilde{Z}$ and $\widetilde{\nabla V}$ denote the extensions of $Z$ and $\nabla V$ to the ambient space $\mathbb{R}^{q}$.

Proof This follows from the chain rule for the tension field of composite maps, that is

$$
\Delta(\iota \circ \phi)=\mathrm{d} \iota(\tau(\phi))+\operatorname{Tr} \nabla \mathrm{d} \pi(\mathrm{d} \phi, \mathrm{d} \phi) .
$$

The homomorphism $Z$ and $\nabla V$ can be extended to the ambient space by projecting to a tubular neighborhood, for more details see [18, p. 463] and [12, p. 557].

\section{Existence results via the heat flow}

In this section, we derive an existence result for critical points of (2.1) by the heat-flow method. In order to achieve this result, we will assume that $\Omega$ is exact such that we have a variational structure that enables us to derive the necessary estimates for convergence of the gradient flow. A similar approach for geodesics coupled to a magnetic field was performed in [5].

In order to control the non-linearities arising from the two-form, we will have to restrict to target spaces with negative sectional curvature. A similar idea has been used in [27] for the heat flow of the prescribed mean curvature equation. More precisely, we will use the negative curvature of the target to control the non-linearities arising from the two-form potential.

For a general introduction to harmonic maps and their heat flows see the book [19].

The gradient flow of the functional (2.1) is given by

$$
\frac{\partial \phi_{t}}{\partial t}(x, t)=\left(\tau\left(\phi_{t}\right)-Z\left(\mathrm{~d} \phi_{t}\left(e_{1}\right) \wedge \mathrm{d} \phi_{t}\left(e_{2}\right)\right)-R \nabla V\left(\phi_{t}\right)\right)(x, t),
$$

where $\phi_{t}: M \times[0, T) \rightarrow N$ and initial data $\phi(x, 0)=\phi_{0}(x)$. To establish the short-time existence of (4.1) we use the Nash embedding theorem to isometrically embed $N$ into $\mathbb{R}^{q}$.

Lemma 4.1 Suppose that $N$ is isometrically embedded into $\mathbb{R}^{q}$. Then (4.1) acquires the form

$$
\frac{\partial u}{\partial t}=\Delta u-\mathbb{I}(\mathrm{d} u, \mathrm{~d} u)-Z\left(\mathrm{~d} u\left(e_{1}\right) \wedge \mathrm{d} u\left(e_{2}\right)\right)-R \nabla V(u),
$$

where $u: M \times[0, T) \rightarrow \mathbb{R}^{q}$.

Proof This follows from Lemma 3.8, we will omit the tildes in order not to blow up the notation.

As a first step, we establish the existence of a short-time solution.

Lemma 4.2 For $\phi_{0} \in C^{2+\alpha}(M, N)$ and $V \in C^{1,1}(N)$ there exists a unique, smooth solution to (4.1) for $t \in\left[0, T_{\max }\right)$.

Proof This can be proven using the Banach fixed point theorem, which requires that the potential $V \in C^{1,1}(N)$. For more details, see [19, Chapter 5].

To extend the solution beyond $T_{\max }$ we will make use of the following Bochner formulae: 
Lemma 4.3 Let $\phi_{t}: M \times\left[0, T_{\max }\right) \rightarrow N$ be a smooth solution of (4.1). Then we have for all $t \in\left[0, T_{\max }\right)$

$$
\begin{aligned}
\frac{\partial}{\partial t} \frac{1}{2}\left|\mathrm{~d} \phi_{t}\right|^{2}= & \Delta \frac{1}{2}\left|\mathrm{~d} \phi_{t}\right|^{2}-\left|\nabla \mathrm{d} \phi_{t}\right|^{2}+\left\langle R^{N}\left(\mathrm{~d} \phi_{t}\left(e_{\alpha}\right), \mathrm{d} \phi_{t}\left(e_{\beta}\right)\right) \mathrm{d} \phi_{t}\left(e_{\alpha}\right), \mathrm{d} \phi_{t}\left(e_{\beta}\right)\right\rangle \\
& -\left\langle\mathrm{d} \phi_{t}\left(R i c^{M}\left(e_{\alpha}\right)\right), \mathrm{d} \phi_{t}\left(e_{\alpha}\right)\right\rangle+\left\langle Z\left(\mathrm{~d} \phi_{t}\left(e_{1}\right) \wedge \mathrm{d} \phi_{t}\left(e_{2}\right)\right), \tau\left(\phi_{t}\right)\right\rangle \\
& -R \operatorname{Hess} V\left(\mathrm{~d} \phi_{t}, \mathrm{~d} \phi_{t}\right)
\end{aligned}
$$

and

$$
\begin{aligned}
\frac{\partial}{\partial t} \frac{1}{2}\left|\frac{\partial \phi_{t}}{\partial t}\right|^{2}= & \Delta \frac{1}{2}\left|\frac{\partial \phi_{t}}{\partial t}\right|^{2}-\left|\nabla \frac{\partial \phi_{t}}{\partial t}\right|^{2}+\left\langle R^{N}\left(\mathrm{~d} \phi_{t}\left(e_{\alpha}\right), \frac{\partial \phi_{t}}{\partial t}\right) \mathrm{d} \phi_{t}\left(e_{\alpha}\right), \frac{\partial \phi_{t}}{\partial t}\right\rangle \\
& -\left\langle\frac{\nabla}{\partial t} Z\left(\mathrm{~d} \phi_{t}\left(e_{1}\right) \wedge \mathrm{d} \phi_{t}\left(e_{2}\right)\right), \frac{\partial \phi_{t}}{\partial t}\right\rangle-R \operatorname{Hess} V\left(\frac{\partial \phi_{t}}{\partial t}, \frac{\partial \phi_{t}}{\partial t}\right) .
\end{aligned}
$$

Proof This follows from the standard Bochner formulas using

$$
\left\langle\nabla Z\left(\mathrm{~d} \phi_{t}\left(e_{1}\right) \wedge \mathrm{d} \phi_{t}\left(e_{2}\right)\right), \mathrm{d} \phi_{t}\right\rangle=-\left\langle Z\left(\mathrm{~d} \phi_{t}\left(e_{1}\right) \wedge \mathrm{d} \phi_{t}\left(e_{2}\right)\right), \tau\left(\phi_{t}\right)\right\rangle .
$$

The Bochner formulae will be the key-tool to achieve long-time existence and convergence of the evolution equation (4.1). However, we have to distinguish between the cases of a compact and a non-compact target.

\subsection{Compact target}

For a compact target manifold $N$ we will prove the following:

Theorem 4.4 Let $(M, h)$ be a closed Riemannian surface and $(N, g)$ be a closed, oriented Riemannian manifold with negative sectional curvature. Moreover, suppose that $\Omega$ is exact, $|B|_{L^{\infty}}<1 / 2, V \in C^{2,1}(N)$ and that the homomorphism $Z$ satisfies

$$
\frac{1}{2}|Z|_{L^{\infty}}^{2} \leq \kappa_{N},
$$

where $\kappa_{N}$ denotes and upper bound on the sectional curvature on $N$. Then $\phi_{t}: M \times[0, \infty) \rightarrow$ $N$ subconverges as $t \rightarrow \infty$ to a harmonic map with scalar and two-form potential $\phi_{\infty}$, which is homotopic to $\phi_{0}$.

We will divide the proof of Theorem 4.4 into several steps.

Lemma 4.5 Let $\phi_{t}: M \times\left[0, T_{\max }\right) \rightarrow N$ be a smooth solution of (4.1) and $V \in C^{2}(N)$. Then for all $t \in\left[0, T_{\max }\right)$ the following inequalities hold:

$$
\frac{\partial}{\partial t} \frac{1}{2}\left|\mathrm{~d} \phi_{t}\right|^{2} \leq \Delta \frac{1}{2}\left|\mathrm{~d} \phi_{t}\right|^{2}+c_{1}\left|\mathrm{~d} \phi_{t}\right|^{2}+\left(\frac{1}{2}|Z|_{L^{\infty}}^{2}-\kappa_{N}\right)\left|\mathrm{d} \phi_{t}\right|^{4}
$$

and

$$
\frac{\partial}{\partial t} \frac{1}{2}\left|\frac{\partial \phi_{t}}{\partial t}\right|^{2} \leq \Delta \frac{1}{2}\left|\frac{\partial \phi_{t}}{\partial t}\right|^{2}+\left(|\nabla Z|_{L^{\infty}}+\frac{1}{4}|Z|_{L^{\infty}}^{2}-\kappa_{N}\right)\left|\mathrm{d} \phi_{t}\right|^{2}\left|\frac{\partial \phi_{t}}{\partial t}\right|^{2}+c_{2}\left|\frac{\partial \phi_{t}}{\partial t}\right|^{2}
$$

with $c_{1}:=\mid$ Ric $\left.\right|_{L^{\infty}}+|R|_{L^{\infty}} \mid$ Hess $\left.V\right|_{L^{\infty}}, c_{2}:=|R|_{L^{\infty}} \mid$ Hess $\left.V\right|_{L^{\infty}}$ and $\kappa_{N}$ denotes an upper bound on the sectional curvature on $N$. 
Proof For the first assertion we consider (4.3) and estimate

$$
\left|\left\langle Z\left(\mathrm{~d} \phi_{t}\left(e_{1}\right) \wedge \mathrm{d} \phi_{t}\left(e_{2}\right)\right), \tau\left(\phi_{t}\right)\right\rangle\right| \leq \sqrt{2}|Z|_{L^{\infty}}\left|\mathrm{d} \phi_{t}\right|^{2}\left|\nabla \mathrm{d} \phi_{t}\right|,
$$

which yields

$$
\left\langle Z\left(\mathrm{~d} \phi_{t}\left(e_{1}\right) \wedge \mathrm{d} \phi_{t}\left(e_{2}\right)\right), \tau\left(\phi_{t}\right)\right\rangle-\left|\nabla \mathrm{d} \phi_{t}\right|^{2} \leq \frac{1}{2}|Z|_{L^{\infty}}^{2}\left|\mathrm{~d} \phi_{t}\right|^{4} .
$$

By assumption $N$ is compact and we can estimate the Hessian of the potential $V(\phi)$ by its maximum yielding the first claim.

For the second claim we consider (4.4) and calculate

$$
\begin{aligned}
\frac{\nabla}{\partial t} Z\left(\mathrm{~d} \phi_{t}\left(e_{1}\right) \wedge \mathrm{d} \phi_{t}\left(e_{2}\right)\right)= & \left(\nabla_{\mathrm{d} \phi_{t}\left(\partial_{t}\right)} Z\right)\left(\mathrm{d} \phi_{t}\left(e_{1}\right) \wedge \mathrm{d} \phi_{t}\left(e_{2}\right)\right)+Z\left(\frac{\nabla}{\partial t} \mathrm{~d} \phi_{t}\left(e_{1}\right) \wedge \mathrm{d} \phi_{t}\left(e_{2}\right)\right) \\
& +Z\left(\mathrm{~d} \phi_{t}\left(e_{1}\right) \wedge \frac{\nabla}{\partial t} \mathrm{~d} \phi_{t}\left(e_{2}\right)\right)
\end{aligned}
$$

which gives

$$
\left\langle\frac{\nabla}{\partial t} Z\left(\mathrm{~d} \phi_{t}\left(e_{1}\right) \wedge \mathrm{d} \phi_{t}\left(e_{2}\right)\right), \frac{\partial \phi_{t}}{\partial t}\right\rangle \leq|\nabla Z|_{L^{\infty}}\left|\mathrm{d} \phi_{t}\right|^{2}\left|\frac{\partial \phi_{t}}{\partial t}\right|^{2}+|Z|_{L^{\infty}}\left|\frac{\nabla}{\partial t} \mathrm{~d} \phi_{t}\right|\left|\mathrm{d} \phi_{t}\right|\left|\frac{\partial \phi_{t}}{\partial t}\right| .
$$

The result follows by applying Young's inequality to the last term on the right hand side.

Via the maximum principle we thus obtain the following:

Corollary 4.6 Let $\phi_{t}: M \times\left[0, T_{\max }\right) \rightarrow N$ be a smooth solution of (4.1). If $\frac{1}{2}|Z|_{L^{\infty}}^{2} \leq \kappa^{N}$ then for all $t \in\left[0, T_{\max }\right)$ the following estimates hold:

$$
\begin{aligned}
\left|\mathrm{d} \phi_{t}\right|^{2} & \leq\left|\mathrm{d} \phi_{0}\right|^{2} e^{2 c_{1} t}, \\
\left|\frac{\partial \phi_{t}}{\partial t}\right|^{2} & \leq\left|\frac{\partial \phi_{0}}{\partial t}\right|^{2} e^{\frac{|\nabla Z|_{L} \infty\left|\mathrm{d} \phi_{0}\right|^{2}}{c_{1}} e^{2 c_{1} t}+c_{2} t} .
\end{aligned}
$$

Proof Using the assumptions the first statement follows by applying the maximum principle to (4.5). For the second statement, we apply the maximum principle to (4.6) using the bound (4.7).

Lemma 4.7 Let $u: M \times\left[0, T_{\max }\right) \rightarrow \mathbb{R}^{q}$ be a smooth solution of (4.2). If $\frac{1}{2}|Z|_{L^{\infty}}^{2} \leq \kappa^{N}$ then the following inequality holds

$$
|u(\cdot, t)|_{C^{2+\alpha}(M, N)}+\left|\frac{\partial u}{\partial t}(\cdot, t)\right|_{C^{\alpha}(M, N)} \leq C,
$$

where the constant $C$ depends on $M, N, Z, \nabla Z, V,\left|\mathrm{~d} \phi_{0}\right|$ and $T_{\max }$.

Proof Interpreting (4.2) as an elliptic equation we may apply elliptic Schauder theory ([16, p. 79]) and get that $u \in C^{1+\alpha}(M, N)$ by the bounds (4.7) and (4.8). Making use of the regularity gained from elliptic Schauder theory we interpret (4.2) as a parabolic equation. The result then follows by application of parabolic Schauder theory ([16, p. 79]).

Lemma 4.8 Let $u, v: M \times\left[0, T_{\max }\right) \rightarrow \mathbb{R}^{q}$ be smooth solutions of (4.2). If $\frac{1}{2}|Z|_{L^{\infty}}^{2} \leq \kappa^{N}$ then the following inequality holds for all $t \in\left[0, T_{\max }\right)$

$$
\left|u_{t}-v_{t}\right|^{2} \leq\left|u_{0}-v_{0}\right|^{2} e^{C t}
$$

for some positive constant $C$. In particular, if $u_{0}=v_{0}$ then $u_{t}=v_{t}$ for all $t \in\left[0, T_{\max }\right)$. 
Proof In the following $C$ will denote a universal constant that may change from line to line. We set $h:=\frac{1}{2}|u-v|^{2}$. By projecting to a tubular neighborhood II $(\mathrm{d} u, \mathrm{~d} u), Z\left(\mathrm{~d} u\left(e_{1}\right) \wedge \mathrm{d} u\left(e_{2}\right)\right)$ and $\nabla V(u)$ can be thought of as vector-valued functions in $\mathbb{R}^{q}$, for more details see [21, p. 132] and also [12, p. 557]. Exploiting this fact a direct computation yields

$$
\begin{aligned}
\frac{\partial h}{\partial t}= & \Delta h-|\mathrm{d} h|^{2}-\left\langle\mathbb{I}_{u}(\mathrm{~d} u, \mathrm{~d} u)-\mathbb{I}_{v}(\mathrm{~d} v, \mathrm{~d} v), h\right\rangle \\
& -\left\langle Z_{u}\left(\mathrm{~d} u\left(e_{1}\right) \wedge \mathrm{d} u\left(e_{2}\right)\right)-Z_{v}\left(\mathrm{~d} v\left(e_{1}\right) \wedge \mathrm{d} v\left(e_{2}\right)\right), h\right\rangle-R\langle\nabla V(u)-\nabla V(v), h\rangle .
\end{aligned}
$$

Rewriting

$$
\mathbb{I}_{u}(\mathrm{~d} u, \mathrm{~d} u)-\mathbb{I}_{v}(\mathrm{~d} v, \mathrm{~d} v)=\left(\mathbb{I}_{u}-\mathbb{I}_{v}\right)(\mathrm{d} u, \mathrm{~d} u)+\mathbb{I}_{v}(\mathrm{~d} u-\mathrm{d} v, \mathrm{~d} u)+\mathbb{I}_{v}(\mathrm{~d} v, \mathrm{~d} u-\mathrm{d} v)
$$

and applying the bounds (4.7), (4.8) we find

$$
\begin{gathered}
\left|\left\langle\mathbb{I}_{u}(\mathrm{~d} u, \mathrm{~d} u)-\mathbb{I}_{v}(\mathrm{~d} v, \mathrm{~d} v), u-v\right\rangle\right| \leq C\left(|u-v|^{2}+|\mathrm{d} u-\mathrm{d} v||u-v|\right), \\
\left|\left\langle Z_{u}\left(\mathrm{~d} u\left(e_{1}\right) \wedge \mathrm{d} u\left(e_{2}\right)\right)-Z_{v}\left(\mathrm{~d} v\left(e_{1}\right) \wedge \mathrm{d} v\left(e_{2}\right)\right), u-v\right\rangle\right| \leq C\left(|u-v|^{2}+|\mathrm{d} u-\mathrm{d} v||u-v|\right)
\end{gathered}
$$

and the second inequality follows similarly. Moreover, by assumption $V \in C^{2,1}(N)$ and thus

$$
|\langle\nabla V(u)-\nabla V(v), u-v\rangle| \leq C h .
$$

Consequently, by Young's inequality we get

$$
\frac{\partial h}{\partial t} \leq \Delta h+C h
$$

for some positive constant $C$. The result then follows by application of the maximum principle.

Proposition 4.9 Let $\phi_{t}: M \times\left[0, T_{\max }\right) \rightarrow N$ be a smooth solution of (4.1). If $\frac{1}{2}|Z|_{L^{\infty}}^{2} \leq \kappa^{N}$ then there exists a unique smooth solution for all $t \in[0, \infty)$.

Proof This follows from the continuation principle for parabolic partial differential equations. Suppose that there would be a maximal time of existence $T_{f i n}$, then using the estimates (4.7) and (4.8) one can show that we can continue the solution for some small $\delta>0$ up to $T_{f i n}+\delta$ yielding a contradiction. The uniqueness follows from Lemma 4.8.

To achieve convergence of the evolution equation (4.1) we will make use of the following:

Lemma 4.10 Assume that $(M, h)$ is a compact Riemannian manifold. If a function $u(x, t) \geq$ 0 satisfies

$$
\frac{\partial u}{\partial t} \leq \Delta u+C u
$$

and if in addition we have the bound

$$
U(t)=\int_{M} u(x, t) \mathrm{d} M \leq U_{0},
$$

then there exists a uniform bound on

$$
u(x, t) \leq e^{C} K U_{0}
$$

with the constant $K$ depending on $M$. 
Proof A proof can for example be found in [26, p. 284]. For more details on how the constant $C$ in the estimate depends on geometric data, see [16, Lemma 2.3.1].

Proposition 4.11 (Convergence) Let $\phi_{t}: M \times[0, \infty) \rightarrow N$ be a smooth solution of (4.1). If $\Omega$ is exact, $\frac{1}{2}|Z|_{L^{\infty}}^{2} \leq \kappa^{N}$ and $|B|_{L^{\infty}}<1 / 2$ then the evolution equation (4.1) subconverges in $C^{2}(M, N)$ to a harmonic map with scalar and two-form potential $\phi_{\infty}$, which is homotopic to $\phi_{0}$.

Proof Since (4.1) is the negative $L^{2}$ gradient flow of the functional (2.1) we obtain

$$
\int_{M}\left(\frac{1}{2}\left|\mathrm{~d} \phi_{t}\right|^{2}+\phi_{t}^{*} B+R V\left(\phi_{t}\right)\right) \mathrm{d} M+\int_{0}^{\infty} \int_{M}\left|\frac{\partial \phi_{t}}{\partial t}\right|^{2} \mathrm{~d} M \mathrm{~d} t=E\left(\phi_{0}\right) .
$$

Thus, for $|B|_{L^{\infty}}<1 / 2$ we get

$$
\int_{M}\left|\mathrm{~d} \phi_{t}\right|^{2} \mathrm{~d} M+\int_{0}^{\infty} \int_{M}\left|\frac{\partial \phi_{t}}{\partial t}\right|^{2} \mathrm{~d} M \mathrm{~d} t \leq C,
$$

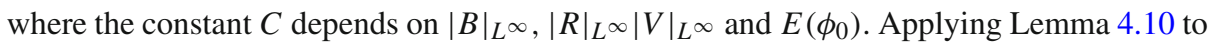
(4.5) and (4.9), we thus obtain a uniform bound on $\left|\mathrm{d} \phi_{t}\right|^{2}$. Inserting this bound into (4.6) we find (for some positive constant $C$ )

$$
\frac{\partial}{\partial t} \frac{1}{2}\left|\frac{\partial \phi_{t}}{\partial t}\right|^{2} \leq \Delta \frac{1}{2}\left|\frac{\partial \phi_{t}}{\partial t}\right|^{2}+C\left|\frac{\partial \phi_{t}}{\partial t}\right|^{2} .
$$

Integrating this equation over $M$ and $t$ from 0 to $\infty$ we get

$$
\int_{M}\left|\frac{\partial \phi_{t}}{\partial t}\right|^{2} \mathrm{~d} M \leq C \int_{0}^{\infty} \int_{M}\left|\frac{\partial \phi_{t}}{\partial t}\right|^{2} \mathrm{~d} M \mathrm{~d} t+\left.\int_{M}\left|\frac{\partial \phi_{t}}{\partial t}\right|^{2}\right|_{t=0} \mathrm{~d} M .
$$

At this point we may again apply Lemma 4.10 to (4.6) and (4.10), which gives

$$
\left|\frac{\partial \phi_{t}}{\partial t}\right|_{L^{\infty}(M \times[0, \infty))}^{2} \leq C .
$$

Now, by (4.9), there exists a sequence $t_{k}$ such that

$$
\int_{M}\left|\frac{\partial \phi_{t}}{\partial t}\left(\cdot, t_{k}\right)\right|^{2} \mathrm{~d} M \rightarrow 0
$$

as $k \rightarrow \infty$. Moreover, by Lemma 4.7 we have

$$
\sup _{k \in \mathbb{N}}\left(\left|\frac{\partial \phi_{t}}{\partial t}\left(\cdot, t_{k}\right)\right|_{C^{\alpha}(M, N)}+\left|\phi_{t_{k}}\right|_{C^{2+\alpha}(M, N)}\right) \leq C .
$$

Then it follows by the Theorem of Arzela and Ascoli that there exists a convergent subsequence, which we will also denote by $t_{k}$. Thus, the family $\phi_{t_{k}}$ subconverges in $C^{2}(M, N)$ to a map $\phi_{\infty}$. Since $\phi_{t}$ depends smoothly on $t$, the limit $\phi_{\infty}$ is homotopic to $\phi_{0}$.

Remark 4.12 Note that we only made use of the globally defined energy (2.1) to obtain convergence of the gradient flow. To achieve long-time existence of (4.1) we do not require a variational structure. 


\subsection{Non-compact target}

In the case that the target manifold $N$ is complete, but non-compact we have to make additional assumptions to control the image of $M$ under the evolution of $\phi$. However, we can use the potential $V(\phi)$ to constrain $\phi_{t}(M)$ to a compact set in $N$. This is similar to the case of the heat flow for harmonic maps with potential [12]. To this end, let $\mathrm{d}_{N}(y)$ denote the Riemannian distance in $N$ from some fixed point $y_{0}$.

Finally, we will prove the following:

Theorem 4.13 Let $(M, h)$ be a closed Riemann surface and $(N, g)$ a complete, oriented Riemannian manifold with negative sectional curvature. Moreover, suppose that $\Omega$ is exact, $|B|_{L^{\infty}}<1 / 2, V \in C^{2,1}(N)$ and $\frac{1}{2}|Z|_{L^{\infty}}^{2} \leq \kappa_{N}$, where $\kappa_{N}$ denotes and upper bound on the sectional curvature on $N$. In addition, assume that the potential $V(\phi)$ satisfies

$$
-R \text { Hess } V(y) \leq-\frac{C}{1+\mathrm{d}_{N}(y)} \quad \text { or } \quad \mid R \text { Hess }\left.V\right|_{L^{\infty}} \leq \frac{\lambda_{1}(M)}{2},
$$

where $\lambda_{1}(M)$ denotes the first eigenvalue of the Laplacian on $M$. Let $\phi_{t}: M \times[0, \infty) \rightarrow N$ be a smooth solution of (4.1). Then $\phi_{t}$ subconverges in $C^{2}(M, N)$ to a harmonic map with scalar and two-form potential, which is homotopic to $\phi_{0}$. Moreover, $\phi_{\infty}$ is minimizing the energy in its homotopy class.

First of all, we make the following observation:

Lemma 4.14 If the three-form $\Omega$ is exact, then $Z \in \Gamma\left(\operatorname{Hom}\left(\Lambda^{2} T^{*} N, T N\right)\right)$ defined via (2.4) is parallel, that is $\nabla Z=0$.

Proof We fix a point $p \in N$ and we extend any tangent vectors $\xi_{1}, \xi_{2}, \eta \in T_{p} N$ to vector fields being synchronous in the point $p$, meaning that

$$
\nabla_{V} \xi_{1}=\nabla_{V} \xi_{2}=\nabla_{V} \eta=0
$$

for all tangent vectors $V \in T_{p} N$. Then using that $\Omega=\mathrm{d} B$ is exact, we find from (2.4)

$$
\mathrm{d} B\left(\xi_{1}, \xi_{2}, \eta\right)=\left\langle Z\left(\xi_{1} \wedge \xi_{2}\right), \eta\right\rangle
$$

By differentiation we then obtain

$$
0=\left\langle\nabla Z\left(\xi_{1} \wedge \xi_{2}\right), \eta\right\rangle
$$

which proves the claim.

As a next step we show how we can use the Hessian of the scalar potential $V(\phi)$ to constrain $\phi_{t}(M)$ to a compact set. This idea has already been applied in the study of the heat flow for harmonic maps with potential, see [12, Proposition 2].

Lemma 4.15 Let $(M, h)$ be a closed Riemann surface and $(N, g)$ a complete Riemannian manifold. Suppose that $\Omega$ is exact and that $\frac{1}{2}|Z|_{L^{\infty}}^{2} \leq \kappa_{N}$. Moreover, assume that the potential $V(\phi)$ satisfies

$$
-R \operatorname{Hess} V(y) \leq-\frac{C}{1+\mathrm{d}_{N}(y)} .
$$

Then there exists a compact set $K \subset N$ such that $\phi_{t}(M) \subset K$ as long as the solution of (4.1) exists. 
Proof Let $\mathrm{d}_{N}$ be the distance function from some point $y_{0}$ in $N$. We set

$$
f(t):=\sup _{x \in M}\left|\frac{\partial \phi_{t}}{\partial t}\right| .
$$

It follows directly that

$$
\mathrm{d}_{N}\left(\phi_{t}\right) \leq C+\int_{0}^{t} f(s) \mathrm{d} s .
$$

Making use of the assumptions on the Hessian of the potential (4.11), the homomorphism $Z$ and the sectional curvature $K^{N}$, we obtain from (4.6) by an argument similar to the compact case that

$$
\frac{\partial}{\partial t}\left|\frac{\partial \phi_{t}}{\partial t}\right|^{2} \leq \Delta\left|\frac{\partial \phi_{t}}{\partial t}\right|^{2}-\frac{C}{1+\int_{0}^{t} f(s) \mathrm{d} s}\left|\frac{\partial \phi_{t}}{\partial t}\right|^{2} .
$$

Then, by the maximum principle, we find

$$
f(t) \leq f(0) \exp \left(-\frac{C}{2} \int_{0}^{t} \frac{1}{1+\int_{0}^{\tau} f(s) \mathrm{d} s} \mathrm{~d} \tau\right) .
$$

We can deduce that

$$
\int_{0}^{t} f(s) \mathrm{d} s \leq C
$$

which gives

$$
f(t) \leq f(0) e^{-C t}
$$

Hence, we find that $\mathrm{d}_{N}(y) \leq C$. This proves that there exists a compact set $K \subset N$ such that $\phi_{t}(M) \subset K$ for all $t \in\left[0, T_{\max }\right)$.

As noted in [9, Proposition 2.4], one can also constrain $\phi_{t}(M)$ to a compact set if the maximum of the Hessian of the potential $V(\phi)$ is bounded by the first eigenvalue of the Laplacian on $M$. This idea can also be applied here:

Lemma 4.16 Let $(M, h)$ be a closed Riemann surface and $(N, g)$ a complete, oriented Riemannian manifold. Suppose that $\Omega$ is exact and that $\frac{1}{2}|Z|_{L^{\infty}}^{2} \leq \kappa_{N}$. Moreover, assume that the potential $V(\phi)$ satisfies

$$
\mid R \text { Hess }\left.V\right|_{L^{\infty}} \leq \frac{\lambda_{1}(M)}{2},
$$

where $\lambda_{1}(M)$ denotes the first eigenvalue of the Laplacian on $M$. Then there exists a compact set $K \subset N$ such that $\phi_{t}(M) \subset K$ as long as the solution of (4.1) exists.

Proof Using (4.4) we derive the following inequality

$$
\begin{aligned}
\frac{\partial}{\partial t} \frac{1}{2} \int_{M}\left|\frac{\partial \phi_{t}}{\partial t}\right|^{2} \mathrm{~d} M \leq & \int_{M}\left(-\left|\nabla \frac{\partial \phi_{t}}{\partial t}\right|^{2}-\kappa_{N}\left|\mathrm{~d} \phi_{t}\right|^{2}\left|\frac{\partial \phi_{t}}{\partial t}\right|^{2}+|Z|_{L^{\infty}}\left|\nabla \frac{\partial \phi_{t}}{\partial t}\right|\left|\mathrm{d} \phi_{t}\right|\left|\frac{\partial \phi_{t}}{\partial t}\right|\right. \\
& \left.-R \operatorname{Hess} V\left(\frac{\partial \phi_{t}}{\partial t}, \frac{\partial \phi_{t}}{\partial t}\right)\right) \mathrm{d} M \\
\leq & \int_{M}\left(-\frac{1}{2}\left|\nabla \frac{\partial \phi_{t}}{\partial t}\right|^{2}+|R \operatorname{Hess} V|_{L^{\infty}}\left|\frac{\partial \phi_{t}}{\partial t}\right|^{2}\right) \mathrm{d} M,
\end{aligned}
$$


where we used Young's inequality and the assumptions on the homomorphism $Z$ and the sectional curvature $K^{N}$ in the last step. By the Kato inequality $|\nabla f|^{2} \geq|\nabla| f||^{2}$ for a function $f: M \rightarrow \mathbb{R}$ and the Poincaré inequality on $M$ we obtain

$$
\int_{M}|\nabla f|^{2} \mathrm{~d} M \geq \lambda_{1}(M) \int_{M} f^{2} \mathrm{~d} M
$$

Applying this inequality we find

$$
\frac{\partial}{\partial t} \frac{1}{2} \int_{M}\left|\frac{\partial \phi_{t}}{\partial t}\right|^{2} \mathrm{~d} M \leq\left(|R \operatorname{Hess} V|_{L^{\infty}}-\frac{\lambda_{1}(M)}{2}\right) \int_{M}\left|\frac{\partial \phi_{t}}{\partial t}\right|^{2} \mathrm{~d} M
$$

yielding

$$
\int_{M}\left|\frac{\partial \phi_{t}}{\partial t}\right|^{2} \mathrm{~d} M \leq C_{0} e^{\left(2|R \operatorname{Hess} V|_{L} \infty-\lambda_{1}(M)\right) t} .
$$

Using the assumption on $\mid R$ Hess $\left.V\right|_{L^{\infty}}$ and applying Lemma 4.10 to (4.4), we obtain a uniform pointwise bound on $\left|\frac{\partial \phi_{t}}{\partial t}\right|$. By the same argument as in the previous Lemma this yields the claim.

By making use of the previous Lemmata we thus find

Lemma 4.17 Let $(M, h)$ be a closed Riemann surface and $(N, g)$ a complete, oriented Riemannian manifold. Moreover, suppose that $\Omega$ is exact, $|B|_{L^{\infty}}<1 / 2, V \in C^{2,1}(N)$ and $\frac{1}{2}|Z|_{L^{\infty}}^{2} \leq \kappa_{N}$. In addition, assume that the potential $V(\phi)$ satisfies

$$
-R \operatorname{Hess} V(y) \leq-\frac{C}{1+\mathrm{d}_{N}(y)} \quad \text { or } \quad|R \operatorname{Hess} V|_{L^{\infty}} \leq \frac{\lambda_{1}(M)}{2},
$$

where $\lambda_{1}(M)$ denotes the first eigenvalue of the Laplacian on $M$. Let $\phi_{t}: M \times[0, \infty) \rightarrow N$ be a smooth solution of (4.1). Then $\phi_{t}$ converges in $C^{2}(M, N)$ to a harmonic map with scalar and two-form potential, which is homotopic to $\phi_{0}$.

Proof By the previous Lemmata we know that $\phi_{t}(M)$ stays inside a compact set $K$. Thus, by Theorem 4.4 there exists a sequence $t_{k}$ such that $\phi_{t_{k}}$ converges to a harmonic map with scalar and two-form potential. Moreover, we have

$$
\mathrm{d}_{N}\left(\phi_{t}, \phi_{\infty}\right) \leq \mathrm{d}_{N}\left(\phi_{t}, \phi_{t_{k}}\right)+\mathrm{d}_{N}\left(\phi_{t_{k}}, \phi_{\infty}\right) .
$$

We know that

$$
\mathrm{d}_{N}\left(\phi_{t}, \phi_{t_{k}}\right) \leq \int_{t_{k}}^{t}\left|\frac{\partial \phi_{s}}{\partial s}\right| \mathrm{d} s \leq C \int_{t_{k}}^{t} e^{-C s} \mathrm{~d} s \rightarrow 0
$$

as both $k, t \rightarrow \infty$, which proves the claim.

Remark 4.18 In the case of the heat flow for harmonic maps with potential to a non-compact target with a concave potential the limit $\phi_{\infty}$ is trivial, see [12, p. 564]. This statement relies on the Bochner formula (3.2), due to the presence of the two-form potential we cannot draw the same conclusion here.

Remark 4.19 If we would only require an upper bound on $R$ Hess $V$ in $N$ then this would be enough to establish long-time existence of (4.1). Consequently, to achieve convergence of (4.1) we need the potential $R V(\phi)$ to constrain $\phi_{t}(M)$ to a compact set. 
Remark 4.20 Using the extrinsic version of the evolution equation (4.2) we can apply the maximum principle to bound the image of $\phi_{t}(M)$. This idea was already used for related geometric flows: a similar criterion as below for the heat flow of harmonic maps with potential is given in [12, Proposition 2]. For the heat flow of the prescribed mean curvature equation in $\mathbb{R}^{3}$ the same idea is used in Proposition 3.2 in [23].

By a direct calculation using (4.2) we obtain

$$
\begin{aligned}
\frac{\partial}{\partial t}|u|^{2} & =\Delta|u|^{2}-2|\mathrm{~d} u|^{2}+\left\langle u, \mathbb{I}(\mathrm{d} u, \mathrm{~d} u)+Z\left(\mathrm{~d} u\left(e_{1}\right) \wedge \mathrm{d} u\left(e_{2}\right)\right)\right\rangle-R\langle u, \nabla V(u)\rangle \\
& \leq \Delta|u|^{2}-2|\mathrm{~d} u|^{2}\left(1-\frac{|u|}{2}\left(|\mathbb{I}|_{L^{\infty}}+|Z|_{L^{\infty}}\right)\right)-R\langle u, \nabla V(u)\rangle .
\end{aligned}
$$

Hence, if we can guarantee that

$$
\frac{|u|}{2}\left(|\mathbb{I}|_{L^{\infty}}+|Z|_{L^{\infty}}\right) \leq 1, \quad R\langle u, \nabla V(u)\rangle>0
$$

at $t=0$, then by the maximum principle we get a bound on $|u|^{2}$ as long as the solution exists. However, the second condition on the potential $V(u)$ is hard to ensure.

\subsection{Minimizing the energy}

In this section, we briefly discuss if the limiting map constructed in Theorem 4.13 is minimizing energy in its homotopy class.

Lemma 4.21 Under the assumptions of Theorem 4.13 the limiting map $\phi_{\infty}$ is minimizing energy in its homotopy class.

Proof We use the formula for the second variation (2.6) to show that the limit $\phi_{\infty}$ obtained in Theorem 4.13 is minimizing the energy in its homotopy class. Let $\phi_{1}, \phi_{2}: M \rightarrow N$ be two smooth maps and let $\Phi: M \times[0,1]$ be a geodesic homotopy between $\phi_{1}$ and $\phi_{2}$, that is $\Phi(\cdot, 0)=\phi_{1}$ and $\Phi(\cdot, 1)=\phi_{2}$. Moreover, assume that for any $x \in M$ the map $\Phi(x, \cdot)$ is a geodesic, when $\phi_{1}$ is a harmonic map with scalar and two-form potential. Using the formula for the second variation (2.6), we find the following inequality

$$
\begin{aligned}
E\left(\phi_{1}\right)-E\left(\phi_{2}\right)= & \int_{0}^{1} d \sigma \int_{0}^{\sigma}\left(\left|\nabla \frac{\partial \Phi}{\partial s}\right|^{2}+\kappa_{N}|\mathrm{~d} \phi|^{2}\left|\frac{\partial \Phi}{\partial s}\right|^{2}-|Z|_{L^{\infty}}|\mathrm{d} \phi|\left|\nabla \frac{\partial \Phi}{\partial s}\right|\left|\frac{\partial \Phi}{\partial s}\right|\right. \\
& \left.-R \operatorname{Hess} V\left(\frac{\partial \Phi}{\partial s}, \frac{\partial \Phi}{\partial s}\right)\right) \mathrm{d} s \\
\geq & \int_{0}^{1} d \sigma \int_{0}^{\sigma}\left(\frac{1}{2}\left|\nabla \frac{\partial \Phi}{\partial s}\right|^{2}+\left(\kappa^{N}-\frac{1}{2}|Z|_{L^{\infty}}^{2}\right)|\mathrm{d} \phi|^{2}\left|\frac{\partial \Phi}{\partial s}\right|^{2}\right. \\
& \left.-R \operatorname{Hess} V\left(\frac{\partial \Phi}{\partial s}, \frac{\partial \Phi}{\partial s}\right)\right) \mathrm{d} s \\
> & 0 .
\end{aligned}
$$

Thus, under the assumptions of the Lemma $\phi_{1}$ is an energy minimizer in its homotopy class.

Remark 4.22 Let $\phi_{t}: M \times[0, \infty) \rightarrow N$ be a smooth solution of (4.1). Under the assumptions of Theorem 4.13 the energy $E(\phi(t))$ is a convex function of $t$, which follows by a direct calculation. 
Exploiting the convexity of the energy $E(\phi(t))$ with respect to $t$ we can prove the following uniqueness Theorem, which is very similar to the case of Hartman's theorem for harmonic maps [15].

Proposition 4.23 Under the assumptions of Theorem 4.13 the limit $\phi_{\infty}$ is independent of the chosen subsequence $t_{k}$.

Proof The proof is the same as in the case of harmonic maps and we omit it here.

Acknowledgements Open access funding provided by [TU Wien (TUW)].

Open Access This article is distributed under the terms of the Creative Commons Attribution 4.0 International License (http://creativecommons.org/licenses/by/4.0/), which permits unrestricted use, distribution, and reproduction in any medium, provided you give appropriate credit to the original author(s) and the source, provide a link to the Creative Commons license, and indicate if changes were made.

\section{References}

1. Aronszajn, N.: A unique continuation theorem for solutions of elliptic partial differential equations or inequalities of second order. J. Math. Pures Appl. 9(36), 235-249 (1957)

2. Baird, P.: Stress-energy tensors and the Lichnerowicz Laplacian. J. Geom. Phys. 58(10), 1329-1342 (2008)

3. Branding, V.: Dirac-harmonic maps with torsion. Commun. Contemp. Math. 18(4) (2016)

4. Branding, V.: Magnetic Dirac-harmonic maps. Anal. Math. Phys. 5(1), 23-37 (2015)

5. Branding, V., Hanisch, F. Magnetic geodesics via the heat flow. (2014). arxiv:1411.6848

6. Chen, Q.: Harmonic maps with potential from complete manifolds. Chin. Sci. Bull. 43(21), 1780-1786 (1998)

7. Chen, Q.: Liouville theorem for harmonic maps with potential. Manuscripta Math. 95(4), 507-517 (1998)

8. Chen, Q.: Maximum principles, uniqueness and existence for harmonic maps with potential and LandauLifshitz equations. Calc. Var. Partial Differ. Equ. 8(2), 91-107 (1999)

9. Chen, Q., Zhou, Z.-R.: Heat flows of harmonic maps with potential into manifolds with nonpositive curvature. Arch. Math. (Basel) 80(2), 216-224 (2003)

10. Eells Jr., J., Sampson, J.H.: Harmonic mappings of Riemannian manifolds. Am. J. Math. 86, 109-160 (1964)

11. Fardoun, A., Ratto, A.: Harmonic maps with potential. Calc. Var. Partial Differ. Equ. 5(2), 183-197 (1997)

12. Fardoun, A., Ratto, A., Regbaoui, R.: On the heat flow for harmonic maps with potential. Ann. Global Anal. Geom. 18(6), 555-567 (2000)

13. Fuchs, J., Nikolaus, T., Schweigert, C., Waldorf, K.: Bundle gerbes and surface holonomy. In: European Congress of Mathematics, pp. 167-195. Eur. Math. Soc., Zürich (2010)

14. Grüter, M.: Conformally invariant variational integrals and the removability of isolated singularities. Manuscripta Math. 47(1-3), 85-104 (1984)

15. Hartman, P.: On homotopic harmonic maps. Can. J. Math. 19, 673-687 (1967)

16. Jost, J.: Nonlinear methods in Riemannian and Kählerian geometry. DMV Seminar, vol. 10. Birkhäuser Verlag, Basel (1988)

17. Koh, D.: The evolution equation for closed magnetic geodesics. Dissertation/PhD-thesis, Universitätsverlag Potsdam (2008)

18. Koh, D.: On the evolution equation for magnetic geodesics. Calc. Var. Partial Differ. Equ. 36(3), 453-480 (2009)

19. Lin, F., Wang, C.: The Analysis of Harmonic Maps and Their Heat Flows. World Scientific Publishing Co., Pte. Ltd., Hackensack (2008)

20. Lin, H., Yang, G., Ren, Y., Chong, T.: Monotonicity formulae and Liouville theorems of harmonic maps with potential. J. Geom. Phys. 62(9), 1939-1948 (2012)

21. Nishikawa, S.: Variational Problems in Geometry, Volume 205 of Translations of Mathematical Monographs. American Mathematical Society, Providence (2002). Translated from the 1998 Japanese original by Kinetsu Abe, Iwanami Series in Modern Mathematics

22. Polchinski, J.: String Theory. Vol. I. Cambridge Monographs on Mathematical Physics. Cambridge University Press, Cambridge (2005). An introduction to the bosonic string, Reprint of the 2003 edition 
23. Rey, O.: Heat flow for the equation of surfaces with prescribed mean curvature. Math. Ann. 291(1), 123-146 (1991)

24. Rudolph, G., Schmidt, M.: Differential Geometry and Mathematical Physics. Part I. Theoretical and Mathematical Physics. Springer, Dordrecht (2013). Manifolds, Lie groups and Hamiltonian systems

25. Sampson, J.H.: Some properties and applications of harmonic mappings. Ann. Sci. École Norm. Sup. (4) 11(2), 211-228 (1978)

26. Taylor, M.E.: Partial Differential Equations III. Nonlinear Equations, Volume 117 of Applied Mathematical Sciences, 2nd edn. Springer, New York (2011)

27. Toda, M.: On the existence of $H$-surfaces into Riemannian manifolds. Calc. Var. Partial Differ. Equ. 5(1), 55-83 (1997)

28. Xin, Y.: Geometry of Harmonic Maps. Progress in Nonlinear Differential Equations and Their Applications, vol. 23. Birkhäuser Boston Inc., Boston (1996) 